\title{
CAS and CEA in the Treatment of Severe Internal Carotid Artery Stenosis
}

\author{
Ling Yao' ${ }^{1}$ Jing Yi' ${ }^{1}$, Lixin $\mathrm{Xu}^{1}$, Jun Wen², Siwei Que ${ }^{1 *}$ \\ 1Department of Neurosurgery, The First People's Hospital of Changde City, Changde 415000, Hunan Province, China \\ ${ }^{2}$ Department of Neurology, The First People's Hospital of Changde City, Changde 415000, Hunan Province, China \\ *Corresponding author: Siwei Que, quesiwei@sina.com
}

\begin{abstract}
Objective: To explore the clinical value of carotid artery stent implantation (CAS) and carotid endarterectomy (CEA) in the treatment of patients with severe internal carotid artery stenosis. Methods: 88 patients with severe carotid artery stenosis who underwent CAS and CEA in the First People's Hospital of Changde City (hereafter referred as "our hospital") from January 2018 to December 2020 were selected as the research objects and divided into CAS group $(n=43)$ and CEA group $(\mathrm{n}=45)$. To understand the clinical application value and feasibility of the two surgical schemes by comparing the general situation, cerebral blood flow, MMSE scale, MOCA scale score and serum miR-145, IGF1R levels of the two surgical schemes. Conclusions: CAS and CEA in the treatment of patients with severe internal carotid artery stenosis, have good curative effect, can effectively improve the patient's cerebral blood flow, regulate serum miR-145, IGF1R levels, promote the recovery of cognitive function, but relatively speaking, the incidence of stroke and hypotension after CAS is higher, and the incidence of hypertension after CEA is higher.
\end{abstract}

Keywords: Severe stenosis of internal carotid artery; Carotid artery stenting (CAS); Carotid endarterectomy (CEA); Cerebral blood flow; miR-145

Publication date: July 2021; Online publication: July 31, 2021

\section{Introduction}

As the main blood supply vessel of human brain, carotid artery mainly undertakes the task of transporting blood for human head, face and neck. Combined with the research results of clinical cerebrovascular diseases, the main cause of insufficient blood supply to the brain and face and cerebral infarction in many patients is caused by carotid artery stenosis. In the treatment of patients with carotid artery stenosis, the commonly used treatment options are drug conservative treatment and surgical treatment. For patients with mild carotid artery stenosis, drug control therapy is generally used, but this treatment method will not cause trauma to patients, but the risk of cerebral ischemia is higher. For patients with severe carotid stenosis, surgical treatment is generally recommended. With the increasing investment in China's medical and health undertakings in recent years, all kinds of new medical equipment and treatment technologies have been developed and achieved ideal results in clinical application. Among them, carotid artery stenting (CAS) and carotid endarterectomy (CEA) are the two most commonly used surgical methods in the treatment of severe carotid stenosis. However, in clinical application the two surgical schemes have obvious curative effect and obvious difference. Therefore, it is of great significance to focus on CAS and CEA. Combined with clinical trials, 88 patients with carotid artery stenosis treated by CAS and CEA were selected as examples. The clinical indexes of the two groups during and two months after operation were compared, and the exact curative effect and clinical application feasibility of different surgical treatment schemes were analyzed. 


\section{Data and methods}

\subsection{General information}

From January 2018 to December 2020, 88 patients with severe carotid stenosis were treated in our hospital. According to the different surgical treatment methods, 43 CAS patients were divided into CAS group. Method: 45 patients who received CEA surgery were divided into CEA group. There was no significant difference in the general clinical data between the two groups $(P>0.05)$, so as to obtain the approval of the hospital ethics committee. Inclusion criteria: The clinical diagnosis showed severe carotid artery stenosis and good intracranial blood supply; Have CAS or CEA operation conditions; Clear consciousness, able to cooperate with the completion of medical research; They were 47-75 years old; The patient and his family members were informed and signed the informed consent to participate in the study. Exclusion criteria: The degree of asymptomatic coronary artery stenosis was more than 50\%; Complicated hypertension, diabetes and acute infectious diseases. The patients were complicated with cerebral hemorrhage and intracranial tumor and had a history of neurosurgery; There are important organ dysfunction; There was cerebral infarction, but internal carotid artery insufficiency was excluded; They have mental illness or are taking psychoactive drugs.

\subsection{Method}

\subsubsection{CAS group}

The patient was in supine position with right side of head. After local anesthesia, the operation was performed and femoral artery puncture was performed; Selective angiography was performed to understand the location, degree and characteristics of carotid artery stenosis. Guided by path map and guide wire, the stent was placed 1-2cm away from the stenosis, and the stent was released $4 \mathrm{~cm}$ away from the distal part of the stenosis with brain protection decoration; For patients with severe stenosis, balloon should be used for pre dilation, and then the stent should be put into the stent. The stent should be released after accurate placement; Aspirin, clopidogrel and atorvastatin calcium were taken routinely for three months after operation. Clopidogrel was stopped after three months, and aspirin and atorvastatin calcium were taken for a long time.

\subsubsection{CEA group}

The patient was in supine position with right side of head, and the operation was carried out after general anesthesia; The anterior edge of sternocleidomastoid muscle should be selected for the incision. If the lesion site of the patient is on the high side, the upper edge of the incision can turn from the mandibular edge to the posterior and upper part to avoid the injury of the marginal mandibular branch of the facial nerve. The common carotid artery, internal carotid artery and external carotid artery were exposed by incision of skin, subcutaneous tissue and platysma; The common carotid artery, internal carotid artery and external carotid artery were blocked in turn; The common carotid artery and internal carotid artery were cut in turn, the carotid artery intima and plaque were stripped, the intima was longitudinally sutured, the intima was reinforced, and the drainage tube was placed for incision suture; Anticoagulant treatment was given six hours after operation. Aspirin, clopidogrel and atorvastatin calcium were taken 24 hours after operation. Clopidogrel was stopped after three months. Aspirin and atorvastatin calcium were taken orally for a long time

\subsection{Observation index and effect judgment}

(1) The operation time, intraoperative blood loss, postoperative mechanical ventilation time, ICU stay time and hospital stay of the two groups were recorded accurately.

(2) Cerebral blood flow the changes of cerebral blood flow in the two groups before and three months after operation were recorded accurately, and the hemodynamic parameters (relative time to peak 
(RTTP), relative mean transit time (rmtt), relative cerebral blood volume (rCBV), relative cerebral blood flow (rcbe)) were calculated.

(3) Serum miR-145 and IGF1R were collected by fasting. $3 \mathrm{ml}$ venous blood was collected before and three months after operation, and centrifuged for $15 \mathrm{~min}$ after standing at room temperature. The rotation speed was set at 3500r/ min. The upper serum samples were collected and stored in the freezer.

(4) The scores of Mini Mental State Examination (MMSE) and Montreal Cognitive Assessment Scale (MoCA) were compared before and three months after operation to understand the degree of cognitive impairment.

\subsection{Statistical methods}

SPSS 20.0 statistical software was used for data analysis, and enumeration data was expressed as [n (\%)]. $\mathrm{X}^{2}$ test was used for comparison, MMSE and MOCA scale scores were tested as $t$ test, and $(\overline{\mathrm{x}} \pm \mathrm{s})$ was used to represent the difference. $P<0.05$ was considered statistically significant.

\section{Results}

\subsection{Comparison of the general situation of the two groups during perioperative period}

By comparing the operation time, intraoperative blood loss, postoperative mechanical ventilation time, ICU stay time and hospitalization time of the two groups, it was found that there was no significant difference in the perioperative general condition indexes between the two groups $(P>0.05)$, as shown in Table 1.

Table 1. Comparison of perioperative general conditions between the two groups $(\overline{\mathrm{x}} \pm \mathrm{s})$

\begin{tabular}{cccccc}
\hline Group & $\begin{array}{c}\text { Operation time } \\
(\mathbf{m i n})\end{array}$ & $\begin{array}{c}\text { Intraoperative } \\
\text { blood loss }(\mathbf{m}) \mathbf{I}\end{array}$ & $\begin{array}{c}\text { Postoperative } \\
\text { mechanical } \\
\text { ventilation } \\
\text { time (H) }\end{array}$ & $\begin{array}{c}\text { ICU stay } \\
\text { time }(\mathbf{d})\end{array}$ & $\begin{array}{c}\text { Length of } \\
\text { stay (d) }\end{array}$ \\
\hline $\begin{array}{c}\text { CAS group } \\
(\mathrm{n}=43)\end{array}$ & $140.75 \pm 25.05$ & $420.50 \pm 76.04$ & $16.58 \pm 3.93$ & $2.69 \pm 0.58$ & $6.98 \pm 1.22$ \\
$\begin{array}{c}\text { CEA group } \\
(\mathrm{n}=45)\end{array}$ & $139.92 \pm 28.90$ & $426.15 \pm 70.55$ & $16.66 \pm 3.31$ & $2.70 \pm 0.55$ & $7.09 \pm 1.12$ \\
$\mathrm{t}$ & 0.1437 & 0.3615 & 0.1035 & 0.0830 & 0.4409 \\
$P$ & 0.8861 & 0.7186 & 0.9178 & 0.9340 & 0.6604 \\
\hline
\end{tabular}

\subsection{Comparison of cerebral blood flow between the two groups}

The RTTP, rmtt, rCBV, rcbe and other indicators of the two groups at THREE months after operation were significantly lower than those before operation $(P<0.05)$, while the RTTP, rmtt, rCBV and rcbe of the two groups after operation showed no significant difference $(P>0.05)$, as shown in Table 2.

\subsection{Comparison of MMSE and MOCA scores between the two groups}

The scores of MMSE and MOCA in the two groups three months after operation were significantly higher than those before operation $(P<0.05)$, but there was no significant difference in the scores of MMSE and MOCA between the two groups $(P>0.05)$, as shown in Table 3. 
Table 2. Comparison of cerebral blood flow between the two groups ( $\overline{\mathrm{x}} \pm \mathrm{s})$

\begin{tabular}{cccccc}
\hline Group & & $\mathbf{R t t p}(\mathbf{s})$ & $\mathbf{r M T T}(\mathbf{s})$ & $\mathbf{r C B V}(\mathbf{s})$ & $\mathbf{R c b e}(\mathbf{m L})$ \\
\hline & Before operation & $1.36 \pm 0.29$ & $1.29 \pm 0.25$ & $1.09 \pm 0.14$ & $1.09 \pm 0.13$ \\
CAS group $(\mathrm{n}=43)$ & After operation & $1.06 \pm 0.14^{*}$ & $1.05 \pm 0.12^{*}$ & $0.92 \pm 0.10^{*}$ & $0.89 \pm 0.11^{*}$ \\
\hline & Before operation & $1.41 \pm 0.22$ & $1.30 \pm 0.26$ & $1.08 \pm 0.15$ & $1.07 \pm 0.17$ \\
CEA group $(\mathrm{n}=45)$ & After operation & $1.06 \pm 0.17^{*}$ & $1.04 \pm 0.18^{*}$ & $0.91 \pm 0.11^{*}$ & $0.87 \pm 0.13^{*}$
\end{tabular}

Note: Preoperative comparison, $P<0.05$; After operation, $P^{*}$ was greater than 0.05 .

Table 3. Comparison of MMSE and MOCA scores between the two groups ( $\overline{\mathrm{x}} \pm \mathrm{s})$

\begin{tabular}{cccc}
\hline Group & & MMSE & MoCA \\
\hline CAS group $(\mathrm{n}=43)$ & Before operation & $23.15 \pm 2.51$ & $22.97 \pm 2.34$ \\
& After operation & $26.19 \pm 1.96^{*}$ & $25.32 \pm 1.66^{*}$ \\
\hline CEA group $(\mathrm{n}=45)$ & Before operation & $23.21 \pm 2.18$ & $22.92 \pm 2.72$ \\
& After operation & $26.30 \pm 1.89^{*}$ & $25.39 \pm 1.58^{*}$ \\
\hline
\end{tabular}

Note: Preoperative comparison, $\mathrm{P}<0.05$; After operation, $\mathrm{P}^{*}$ was greater than 0.05 .

\subsection{Comparison of serum miR-145 and IGF1R levels between the two groups}

Compared with before operation, the expression of serum miR-145 was significantly increased and IGF1R was significantly decreased in the two groups 3 months after operation $(\mathrm{P}<0.05)$. There was no significant difference in the expression of serum miR-145 and IGF1R between the two groups after operation $(\mathrm{P}>$ 0.05), as shown in Table 4.

Table 4. Comparison of changes in serum miR-145 and IGF1R levels between the two groups ( $\overline{\mathrm{x}} \pm \mathrm{s})$

\begin{tabular}{cccc}
\hline Group & miR-145 & IGFIR(ng/mL) \\
\hline \multirow{2}{*}{ CAS group $(\mathrm{n}=43)$} & Before operation & $0.41 \pm 0.06$ & $3.59 \pm 0.75$ \\
& After operation & $0.89 \pm 0.12^{*}$ & $2.60 \pm 0.39^{*}$ \\
\hline CEA group $(\mathrm{n}=45)$ & Before operation & $0.41 \pm 0.04$ & $3.58 \pm 0.80$ \\
& After operation & $0.89 \pm 0.12^{*}$ & $2.56 \pm 0.47^{*}$ \\
\hline
\end{tabular}

Note: Preoperative comparison, $P<0.05$; After operation, $P^{*}$ was greater than 0.05 . 


\section{Discussion}

At present, the clinical treatment for patients with carotid artery stenosis mainly includes drug treatment and surgical treatment. Although the former will not cause trauma to patients, the prognosis is not ideal. Therefore, many patients and doctors are willing to adopt surgical treatment. With the further improvement of surgical technology, CAS and CEA are considered to be the most effective surgical methods in the treatment of patients with severe carotid artery stenosis. However, due to the limitation of various clinical experimental design standards, inclusion criteria and differences in the level of physicians, there are differences in the efficacy of CEA and CAS to varying degrees.

In this study, combined with clinical trials, by comparing the clinical indicators of patients with CEA and CAS, we found that there was no significant difference between the two groups in perioperative general indicators, and there was no statistical significance; At three months after operation, the cerebral blood flow, MMSE and MOCA scores of the two groups were significantly improved compared with those before operation, which showed that the two methods were helpful to improve the cerebral blood flow and cognitive dysfunction of patients with severe carotid stenosis.

In conclusion, CAS and CEA have similar clinical efficacy in the treatment of patients with severe internal carotid artery stenosis, which can effectively improve the cerebral blood flow, regulate the serum levels of miR-145 and IGF1R, and promote the recovery of cognitive function. However, the incidence of stroke and hypotension after CAS is higher, and the incidence of hypertension after CEA is higher, which needs further research.

\section{Disclosure statement}

The author declares no conflict of interest.

\section{Author contributions}

Ling Yao and Jing Yi both are the first authors and contributed equally to this work.

\section{References}

[1] Wang D, Liang W, Gao Y, 2021, Observation on the Effect of Stent Placement After Small Balloon Dilatation in the Treatment of Severe Stenosis at the Beginning of Internal Carotid Artery. Chinese Journal of Practical Medicine, (06):10.

[2] Hu C, Dong R, Shi F, et al., 2021, Effect of CAS and CEA On Cerebral Blood Flow, Serum miR145 and IGF1R in the Treatment of Severe Internal Carotid Artery Stenosis. Progress of Modern Biomedicine. (03) - 30

[3] Liu Q, Yu C, Jia H, et al., 2021, Effect of Internal Carotid Artery Stenting on Cognitive Impairment in Patients with Severe Internal Carotid Artery Stenosis and Analysis of Related Risk Factors. Chinese Journal of Physicians, 1:20.

[4] Chen C, Yuan X, Han J, et al., 2020, Efficacy and Safety of Staged Stenting in the Treatment of Subtotal Occlusion of Internal Carotid Artery with Contralateral Internal Carotid Artery Stenosis. Chinese Journal of Stroke, (08):20.

[5] Sun Q, Mao M, Yan W, et al., 2020, Effect of Carotid Artery Stenting on Cognitive Function of Patients with Severe Internal Carotid Artery Stenosis. Contemporary Medicine, (07):25.

[6] Yuan H, Qian K, Fu F, et al., 2020, Analysis of Early Curative Effect of Modified Carotid Endarterectomy for Severe Carotid Stenosis. Chinese Journal of Vascular Surgery (Electronic Edition), (06):20. 